\title{
Subjective endpoints in clinical trials: the case for blinded independent central review
}

This article was published in the following Dove Press journal:

Open Access Journal of Clinical Trials

24 September 2013

Number of times this article has been viewed

\section{Richard Walovitch' \\ Bin Yao ${ }^{2}$ \\ Patrick Chokron' \\ Helen Le' \\ Glenn Bubley ${ }^{3}$ \\ 'WorldCare Clinical, LLC, Boston, MA, USA; ${ }^{2}$ Amgen, Inc, Thousand Oaks, CA, USA; ${ }^{3}$ Director of Genitourinary Medical Oncology, Beth Israel Deaconess Medical Center, Boston, MA, USA}

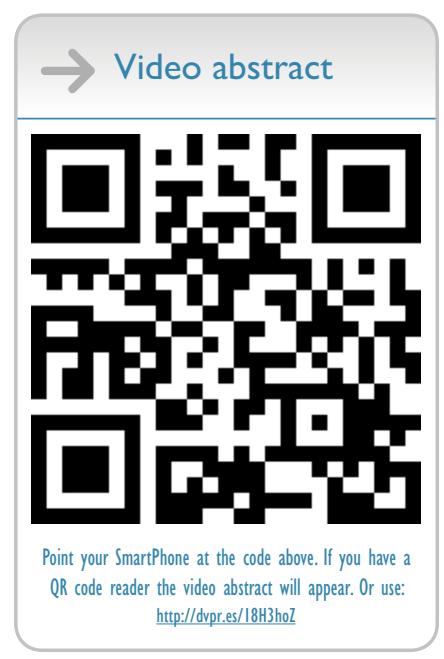

Correspondence: Richard C Walovitch WorldCare Clinical, LLC, 7 Bulfinch Place, PO Box 8908,

Boston, MA 021।4, USA

$\mathrm{Tel}+\mathrm{I} 6175831606$

Fax +I 5133529564

Email rwalovitch@wcclinical.com
Abstract: Primary efficacy and safety endpoints in clinical trials are often subjective assessments made by site personnel. For international confirmatory trials conducted over broad geographic regions and different clinical practice settings, variability in these subjective assessments can be substantial. Centralized endpoint assessment committees (EACs) offer a mechanism through which to reduce assessment bias and potentially increase assessment precision and accuracy, particularly in open-label trials. An overview of regulatory agencies' rationales for an EAC is reviewed. In addition, the two main types of EACs, the blinded independent central review, and the consensus panel are compared. Selection of endpoints for EAC evaluation and design of EAC process to maximize EAC value proposition are also discussed.

Keywords: endpoint assessment committee, FDA, central review, BICR, adjudication, consensus panel

\section{Introduction}

Reducing assessment variability and bias are major considerations when a trial's primary endpoint is subjective. Regulatory agencies have recognized these issues and have recommended that site evaluations are repeated by an independent committee of experts with the assumption that these expert assessments would be less biased and less variable than site assessments. ${ }^{1-4}$ The need for this blinded independent central review (BICR) increases as the trial goes from Phase I to Phase III, with the need being most important for the primary endpoint of confirmatory trials. ${ }^{5}$

BICRs are the default standard for confirmatory trials of imaging agents, ${ }^{6}$ and have been addressed recently in the US Food and Drug Administration's (FDA) guidance document on the use of imaging agents as endpoints. ${ }^{3}$ The position of the agency with regards to the value of these BICRs is well summarized in a FDA guidance document which states “... the centralized process can better provide verifiable and uniform reader training as well as ongoing management of reader performance, ensuring that the process is accurate and that bias and variability are minimized." 3

Although it is clear that the major driver for the use of endpoint assessment committees (EACs) for imaging has been regulatory approvals, the clinical research community has also driven home the importance of these independent assessments particularly as it relates to complex and variable subjective endpoints in large international trials. ${ }^{7-9}$ For example, over 10 years ago, investigators in the PURSUIT (Platelet Glycoprotein IIb/IIIa in Unstable Angina: Receptor Suppression Using Integrilin Therapy) trial evaluated Integrilin in 10,948 patients for the treatment of acute coronary syndrome and concluded that independent adjudication is needed in 
trials that span geographical regions and practice settings. ${ }^{7}$ Furthermore, an independent adjudication process "provides a standard, systematic, independent and unbiased assessment of end-points." ${ }^{\text {77 }}$ This and similar trials were probably the driving forces behind the FDA's guidance that all Phase II and Phase III trials in type 2 diabetes establish an independent endpoint committee to prospectively adjudicate cardiovascular events. ${ }^{10}$

The objective of this review is to highlight the critical roles an EAC can play in the drug development process, explain the different types of EACs, and provide a process map for conducting an EAC. The examples provide broader context and considerations than the views on imaging discussed in the FDA draft guidance document on the use of medical imaging. ${ }^{3}$

\section{Nomenclature}

EAC is the term used consistently throughout this review; however, the term is far from universal. EAC is derived from a 2005 article by Kradjian et al. ${ }^{11}$ In the original article, the authors refer to EAC as the Endpoint Assessment and Adjudication Committee. ${ }^{11}$ Another common acronym used is CEC, which stands for both Clinical Endpoint Committee and Clinical Event Committee. The FDA uses a different acronym, IRC, which is defined as both independent endpoint review committee and independent radiology review. ${ }^{12}$ Regardless of the nomenclature, the concept is the same; an assessment of a critical subjective endpoint performed by experts who are not affiliated with trial sites or the sponsor and are blinded to patient treatment. These committees are often established to independently assess the primary endpoint for Phase III confirmatory trials. The goal of an EAC is to perform an integrated interpretation of subjective clinical data. This can be focused on efficacy or safety endpoints. ${ }^{3}$

EACs are sometimes confused with the more common data safety monitoring boards (DSMBs). Although a DSMB is a committee of experts who are also independent of trial sites and the sponsor, they function much differently than an EAC. As the DSMB acronym implies, its responsibility is to safeguard the safety of the trial participants and to provide reviews of accumulating data to assess benefit and risk of the treatment intervention. DSMBs are often convened at preset time periods during the conduct of a clinical trial. For example, a DSMB may review safety data when the first 20 patients have been treated with three cycles of an experimental agent. In contrast, an EAC generates independent endpoint data for each patient. Table 1 highlights the major differences of these two independent committees.
Table I Comparison of DSMB to EAC

\begin{tabular}{|c|c|c|}
\hline Attribute & DSMB & EAC \\
\hline Membership & $\begin{array}{l}\text { Subspecialty expert } \\
\text { clinicians and statisticians }\end{array}$ & $\begin{array}{l}\text { Subspecialty expert } \\
\text { clinicians }\end{array}$ \\
\hline $\begin{array}{l}\text { Objective of } \\
\text { process }\end{array}$ & $\begin{array}{l}\text { Ongoing benefit risk } \\
\text { assessment of } \\
\text { accumulating trial data }\end{array}$ & $\begin{array}{l}\text { Generate independent, } \\
\text { less biased endpoint } \\
\text { data }\end{array}$ \\
\hline $\begin{array}{l}\text { Types of data } \\
\text { reviewed }\end{array}$ & $\begin{array}{l}\text { Primarily safety, } \\
\text { superiority, or futility } \\
\text { for efficacy; unblinded to } \\
\text { treatment assignment }\end{array}$ & $\begin{array}{l}\text { Eligibility, important } \\
\text { safety and/or efficacy } \\
\text { parameters, patient } \\
\text { outcomes; blinded to } \\
\text { treatment assignment }\end{array}$ \\
\hline $\begin{array}{l}\text { Data review } \\
\text { process }\end{array}$ & $\begin{array}{l}\text { Group sequential review } \\
\text { of multiple patients }\end{array}$ & $\begin{array}{l}\text { Mostly independent } \\
\text { review of each patient }\end{array}$ \\
\hline $\begin{array}{l}\text { Frequency of } \\
\text { data review }\end{array}$ & $\begin{array}{l}\text { Periodic and specified by } \\
\text { protocol based on either } \\
\text { calendar time or event rate }\end{array}$ & $\begin{array}{l}\text { Every patient, and } \\
\text { often continuous }\end{array}$ \\
\hline
\end{tabular}

Abbreviations: DSMB, data safety monitoring board; EAC, endpoint assessment committee.

\section{Types of EACs}

EACs' assessments are often performed using BICRs, consensus assessment reviews, or a combination of both. BICRs are typically performed by a homogenous panel of independent reviewers (IRs). Consensus reviews are performed by a panel of two or more reviewers who often do not have the same background and training. Historically, consensus assessments were the default when EAC data involved multiple disciplines. In an oncology trial, for example, the EAC assessment would have to interpret pathology, radiology, and clinical data. These reads were a combination of paper and digital data presented to a panel for their evaluation of the patient status. More quantitative methodology exists; however, these methods have been primarily developed to determine diagnostic and classification guidelines, ${ }^{13}$ and little, if any, attention is focused on patient data review. For patient data review, the "expert panel", which is based on the nominal group technique, ${ }^{13-15}$ is usually a structured meeting (real or digital) that often consists of large panels of experts (5-12) with one to two rounds of assessments/decisions; after each round, results are analyzed for agreement using predefined rules.

More recently, with the development of sophisticated, fully digital EAC evaluation systems, all the data can be provided electronically. This development greatly facilitates the BICR approach by allowing for multiple concurrent independent assessments of data, followed by an adjudication review triggered programmatically, if needed.

Table 2 compares a pure consensus panel approach to a pure BICR approach. Although many different permutations of these methods can be chosen, including a combination of both approaches, Table 2 illustrates the benefits and 
Table 2 Types of EACs

\begin{tabular}{|c|c|c|}
\hline Attribute of EAC & Consensus & Multireader BICR \\
\hline $\begin{array}{l}\text { Potential for gestalt } \\
\text { understanding of } \\
\text { the patient }\end{array}$ & $\begin{array}{l}\text { Better than BICR, } \\
\text { but could be offset by } \\
\text { groupthink mentality }\end{array}$ & Less than consensus \\
\hline $\begin{array}{l}\text { Member } \\
\text { communication }\end{array}$ & $\begin{array}{l}\text { Multidirectional } \\
\text { discussions with little } \\
\text { structure or control }\end{array}$ & $\begin{array}{l}\text { One-way hierarchical } \\
\text { communication where } \\
\text { results of one or more } \\
\text { reviewers feeds into } \\
\text { next reviewer in a } \\
\text { predefined manner }\end{array}$ \\
\hline Member expertise & Multidisciplinary team & $\begin{array}{l}\text { Homogenous, } \\
\text { subspecialty-trained } \\
\text { clinicians }\end{array}$ \\
\hline Precision & $\begin{array}{l}\text { Single "group" } \\
\text { assessment }\end{array}$ & $\begin{array}{l}\text { Multiple independent } \\
\text { assessments with } \\
\text { adjudication as needed }\end{array}$ \\
\hline $\begin{array}{l}\text { Member training } \\
\text { and testing }\end{array}$ & Impractical & Easy to perform \\
\hline $\begin{array}{l}\text { Logistical } \\
\text { complexity }\end{array}$ & $\begin{array}{l}\text { Complex scheduling } \\
\text { and coordination of } \\
\text { panel members }\end{array}$ & $\begin{array}{l}\text { Complex due to } \\
\text { sequential review } \\
\text { process }\end{array}$ \\
\hline $\begin{array}{l}\text { Operational } \\
\text { complexity }\end{array}$ & $\begin{array}{l}\text { Easy to perform if } \\
\text { nondigital }\end{array}$ & $\begin{array}{l}\text { Complicated and } \\
\text { technically challenging }\end{array}$ \\
\hline
\end{tabular}

Abbreviations: EAC, endpoint assessment committee; BICR, blinded independent central review.

drawbacks of each approach. The advantage of the consensus approach is the ability to synthesize decisions with input from many different experts all focusing on different aspects of the patient's disease status, and thus providing the potential for a gestalt understanding of the patient's status through real-time dialogue. This also avoids the departmentalization experienced in the "silo approach" assessment of the BICR. Unfortunately, this advantage may not often be realized because consensus panels are vulnerable to a "groupthink mentality" where the most popular position, the one articulated by the loudest voice or by the highest-ranking individual, often prevails over the correct answer. ${ }^{16-18}$

The consensus panel usually results in one site independent assessment per patient. In contrast, because a BICR can consist of multiple independent assessments, interreader variability can be analyzed, and thus provide a better understanding of differences in interpretation strategies between EAC members. Understanding of interreader variability can be leveraged in training and testing of the IRs. An additional advantage of a BICR is the ability to selectively add precision to different components of the assessment by utilization of IRs with different levels of expertise. This can result in a cost-effective utilization of experts that cannot be replicated in the consensus panel approach.

Figure 1 illustrates this concept with a workflow example from a complex EAC. In this example, multiple serial and parallel assessments are performed to determine the total tumor burden of patients in a large, double-blind, multicenter, international melanoma trial. This trial consists of four sets of data from different clinical experts, which must be integrated for independent oncology determination in the overall EAC assessment. The need to perform independent assessments

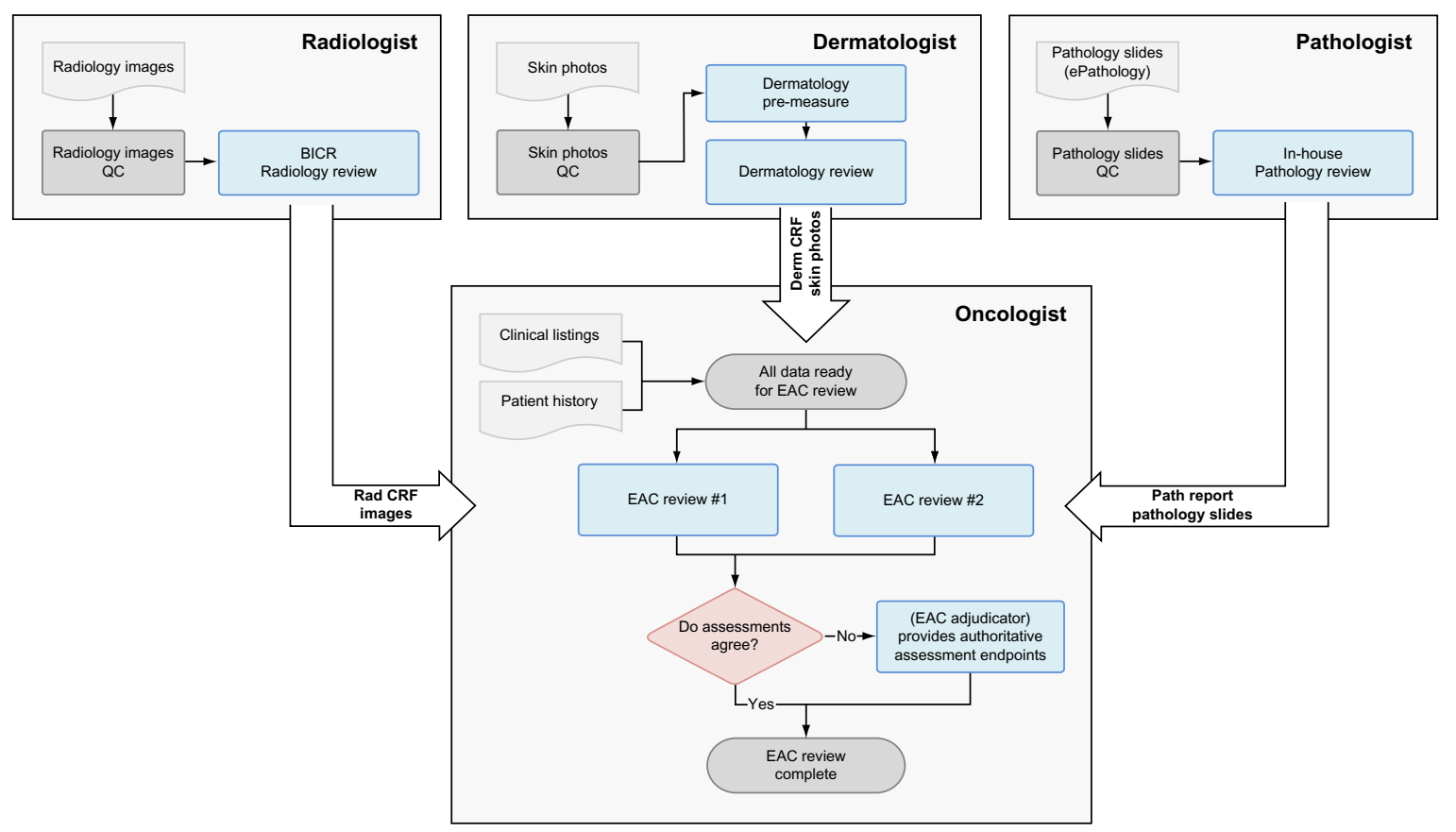

Figure I Melanoma workflows for EAC using BICRs.

Abbreviations: Rad, radiology; BICR, blinded independent central review; Derm, dermatology; QC, quality control; Path, pathology; CRF, case report form; EAC, endpoint assessment committee. 
should be predicated on the potential for bias along with the variability and complexity of the assessment. For both radiology and dermatology assessments, single-read BICRs are performed for the following reasons:

1. Site bias is a concern since treatment-specific adverse experiences may be unblinding the investigator to the treatment arms. ${ }^{4}$

2. The quantification of tumor burden via radiology and dermatology is complex, making it expensive and hard to effectively train trial site personnel.

In addition, it is of value to consider premeasurements when a large number of lesions exist. Premeasurements are performed by less experienced, and therefore less costly, reviewers who annotate the images for the final review by the board-certified, subspecialty-trained IR. Pathology is performed by the site reviewer only since the assessment is binary (melanoma/no melanoma) and precision, accuracy and unblinding of the assessment is less of a concern than with radiology and dermatology. The oncology review of overall tumor burden is the final review and determines the patient's disease status as it relates to the primary efficacy analysis (in this case, for progression or lack of progression at each cycle of therapy). Since a complex and variable assessment will determine study outcomes, the data is independently read by two IRs, and discrepancies are adjudicated by a third IR to decrease variance.

Coupling the multireader paradigm with the ability to train and test blinded IRs provides data that are as standard as possible, thus increasing the validity and integrity of the studies and maximizing the ability to discern smaller effects or differences between groups. This advantage could lead to a decrease in trial sample size. ${ }^{5,19-21}$ For example, a simulation tumor growth model with a progression-free survival endpoint showed that measurement variability resulted in attenuation of the treatment effect (ie, a hazard ratio closer to one) and led to an increase in type 2 error, which could only be compensated by increasing the sample size. ${ }^{19,21}$ A similar model has demonstrated the resultant decrease in sample size expected from the decrease in variance. As a result, BICR may afford cost savings that more than offset the cost of the independent assessment. ${ }^{20}$ When the investigators or sponsors do not choose to alter the sample size based on the advantage of a BICR, a gain in power for the endpoint may be expected with the reduced variability. In addition to the expected reduction in variability, there are situations when the power can be potentially improved by formally incorporating the level of certainty of endpoint assessment into statistical models. ${ }^{22}$
No direct cost comparisons have been done between a site and consensus approach. However, modeling results from a lung cancer study showed that consensus committees may have more panelists than needed and, in many cases, committees need not consist of more than three members since the increase in panelists has little effect on the results. ${ }^{15}$

As the size and complexity of confirmatory trials increase, the challenges involved in managing digital EACs also increase proportionately. From a technical standpoint, the all-digital multireader BICR is more complex than the nondigital or partially digital consensus panel, which is in large part due to its programmatic complexity and hierarchical review structure. Electronic systems have become essential to successfully collect, organize, and present data for IRs in a regulatory controlled environment. The EAC input data, including clinical patient data, medical images, pathology slides, and other test results must be funneled into a coherent central archive. The process starts at investigator sites where electronic data capture is used to record form data. Radiology, dermatology, and/or pathology images are either digitally uploaded or sent via courier to a central archive. Since digital pathology is still in its infancy, most study pathology blocks and glass slides need to be sent to a core laboratory for scanning. Ultimately, all data is converted to digital formats, and quality controlled for completeness and accuracy prior to review by the EAC. Therefore, as discussed above, the design of a digital EAC review system must contend with two key challenges:

1. How to integrate the information to optimize the quality of the review process.

2. How to efficiently manage cases through complex review workflows.

\section{Understanding the input function}

The input function of an EAC is dependent on what data is required to evaluate the question being asked. For many EACs, little or no ambiguity exists about what data needs to be reviewed. For example, in radiology and pathology EACs, the input function is often unequivocal (images and pathology slides that relate to response). In contrast, EACs often rely on multiple sources of information that can affect endpoint assessment including laboratory parameters and physical examination assessments. The EAC determination of patient status is not the same as the practice of medicine; it should be viewed as regulatory science (Table 3 ). The systems used for an EAC assessment will not be able to, and should not, capture all the information that the principal investigator (PI) has, in large part because the 
Table 3 Site versus BICR of patient status

\begin{tabular}{|c|c|c|}
\hline & Site assessment & EAC assessment \\
\hline Dataset & $\begin{array}{l}\text { All available captured; } \\
\text { clinical information and } \\
\text { nondocumented information }\end{array}$ & $\begin{array}{l}\text { Prespecified } \\
\text { minimum dataset }\end{array}$ \\
\hline Data standard & $\begin{array}{l}\text { Institutional policies vary } \\
\text { from site to site }\end{array}$ & $\begin{array}{l}\text { Uniformed } \\
\text { centralized process }\end{array}$ \\
\hline Bias & $\begin{array}{l}\text { Greater potential when the } \\
\text { trial is open label or when } \\
\text { known treatment effects } \\
\text { could lead to potential } \\
\text { unblinding }\end{array}$ & $\begin{array}{l}\text { Less potential } \\
\text { because it is blinded } \\
\text { to treatment } \\
\text { assignment, and BICR } \\
\text { evaluators have no } \\
\text { relationship with } \\
\text { the clinical subject }\end{array}$ \\
\hline $\begin{array}{l}\text { Feedback to } \\
\text { patient/sites }\end{array}$ & $\begin{array}{l}\text { Potential to change patient } \\
\text { management }\end{array}$ & None, in most cases \\
\hline
\end{tabular}

Abbreviations: BICR, blinded independent central review; EAC, endpoint assessment committee.

EAC does not share the same responsibility as the PI with respect to the patient. The objective of the EAC is to make an unbiased and independent assessment of the patient status using standardized criteria based on prespecified data sets. This is in contrast with the treating physician at the site, who may find the practice of medicine in conflict with compliance in clinical trials. ${ }^{23}$ The tradeoff is a more gestalt assessment - and therefore, a potentially more accurate assessment by the site - versus a less biased, potentially less variable (higher interreader reproducibility), and more homogenous interpretation with the BICR. The BICR performed by an EAC is better characterized since the input function is more controlled and training of the EAC on a decision tree algorithm can be much more extensive than what is practical for a large number of clinical investigators in an international multicenter trial.

There are multiple considerations that need to be incorporated into the determination of which data need to be evaluated by the EAC. This assessment should start with an understanding of the accepted clinical criteria for making any endpoint assessments that are made by the EAC. Definitions of progression and response are often addressed by the World Health Organization, FDA, and/or European Medicines Agency. If it is an evolving area and international and/or regulatory guidance is not yet available, consensus panel publications (sponsored by professional clinical societies) can often be instrumental. The input function should be determined by clinical scientists from the EAC who have, at a minimum, the same subspecialty training and clinical experience as the site assessor (for example, an oncologist for cancer study). It is often desirable to use more experienced and more specialized experts (for example, an epileptologist rather than a general neurologist for a study of temporal lobe epilepsy).

\section{Common EAC mistakes}

In our experience, the most common and significant errors with EACs fall into the following categories: unclear designation of EAC endpoints as a result of insufficient prospective documentation of EAC processes; lack of clarity regarding data required for EAC assessment; and operational mistakes that result in potential bias, such as not clearly defining criteria needed for EAC rereads, failing to ensure that the reviewers are independent of the sponsor and site personnel (although this is needed with all blinded independent reviews), and data not being anonymized.

The heterogeneity of EACs and the lack of regulatory guidance are contributing factors to a lack of clarity in the regulatory documentation related to EAC endpoints. The issue is usually not related to defining what the endpoint parameter is (ie, disease progression), but rather defining whose determination of that parameter constitutes the endpoint. For example, protocols and statistical analysis plans (SAPs) may state that the primary endpoint is disease progression, but they may fail to indicate that the EAC's determination of disease progression, not the site's determination, is primary. The general design and statistical concepts that relate to the EAC are included in both the protocol and SAP. Additional details related to the EAC organization and process are best provided in a separate document called a charter. Since most information in a charter (ie, read paradigm, training of reviewers, qualifications of reviewers) does not involve patient management, generating or amending a charter can be done without involving institutional review boards or modifying the protocol, thus making it less of a regulatory and logistical challenge. Depending upon the division of the FDA, the EAC charter may not always be required to be filed with the protocol. However, for imaging studies the FDA encourages "submission of the charter simultaneously with a complete clinical protocol, including the final SAP."3 This approach has been used successfully for multiple EACs in different therapeutic areas, and is clearly outlined in the recent FDA draft imaging guidance documents, which can be used as a process roadmap for many components of an EAC charter. ${ }^{3}$ Specifically, the EAC charter should cover all aspects of the independent assessment, such as the responsibility of the sponsor, contract research organization (CRO), and IRs; qualification for IRs; data required for $\mathrm{EAC}$ review; interpretation guidelines; and data transfer and archiving.

When it is unclear what the data requirements are for an EAC assessment, the trial sponsor often suggests that all 
clinical data be sent to the EAC. This approach is seldom helpful since it creates a number of problems:

1. What does all patient data mean? Is it the whole patient chart? What constitutes clinically relevant information of value that has significant bearing on the endpoint? For example, does the EAC need to know if the patient ate dinner or if the patient feels a little nervous? Perhaps this is the case in assessing a new diet pill, but it is not important for an oncolytic agent.

2. What constitutes source documentation? How can a sponsor determine if some information in the patient files not sent to the EAC should have been sent? Lack of clarity in this area can create tremendous variability in the input function and could introduce bias in the trial conduct. The effect of bias could be a major issue with regulatory agencies since rereading of data can be performed if new but questionable "clinically relevant" data is found. This reread issue has been a major concern of an FDA's Oncologic Drugs Advisory Committee. ${ }^{24}$ This is why, as stated previously, it is essential to determine in advance the data needed to make the assessment and to have it specified in the EAC charter.

Bias is also of concern when ensuring that appropriate documentation and systems are prospectively defined and operational prior to the start of EAC reviews, which demonstrate independence of the central review process from both the site assessments and sponsor influence. A thoughtful and robust EAC charter outlining detailed plans for bias minimization is an important first step. Experience and vigilance are also critical in ensuring proper execution of the plans. In practice, what is more insidious and requires $100 \%$ data verification by the $\mathrm{CRO}$ is assuring that the data reaching the EAC is anonymized. Anonymization of patient identity and treatment arm alone is not sufficient. Information regarding the site location and treating physician's name, which is often on pathology reports or embedded in images, must be removed. When safety data is included, it is often overlooked that there is a need to redact information from the site generated adverse experience narratives. In open-labeled trials, treatment arm information may appear in the narratives, thus requiring careful review and redacting.

\section{Conclusion}

The rapidly increasing cost of bringing a new drug to market is currently estimated at $\$ 1.3$ billion. ${ }^{25}$ The most expensive component of this process is typically a pivotal confirmatory, trial which has the capacity to determine the success or failure of the entire development program. ${ }^{25}$ For pivotal trials that are either open label or have the potential to become unblinded as a result of certain known treatment effects, it is critical to establish the EAC to ensure trial integrity and interpretability of its data. When the primary endpoint involves clinical components beyond imaging, the complexity of the EAC process will increase. Although central reviews using an EAC have become standard requirements for imaging, they are not often required to determine the overall clinical status of the patient. The FDA has commented that the variability in EAC trial design makes it difficult to provide a general guidance document. This, coupled with the lack of a clear understanding of what data needs to be included in the overall assessment process, has historically resulted in limited use of overall patient status EACs in general, and particularly EACs using the BICR approach. Seamless integration of technology and regulatory guidance into the EAC process can help improve consistency, increase reliability, and reduce variability in the assessment of primary efficacy endpoints. It is possible that this may translate into a greater probability of the trial's success. Furthermore, recent trends are more favorable for greater uptake of BICRs in the drug development industry:

1. Lower cost of BICRs as a result of the advance in telemedicine and advanced information systems;

2. An increase in the utilization of inductive inference to understand which data are needed to make an overall patient status assessment, and the integration of these specific data elements into the site case report form; and

3. Advances in regulatory science and better coordination among the stakeholders in clinical trials.

Advances in the aforementioned attributes are expected to continue to accelerate, which should further drive the value proposition for BICRs.

\section{Disclosure}

Richard Walovitch, Patrick Chokron and Helen Le are fulltime employees of WorldCare Clinical, LLC - a company that provides imaging $\mathrm{CRO}$ services. Bin Yao is a full-time employee of Amgen Inc. All of the authors report no other conflicts of interest in this work.

\section{References}

1. European Medicines Agency; Committee for Medicinal Products for Human Use. Appendix 1 to the Guideline on Clinical Evaluation of Diagnostic Agents (CPMP/EWP/1119/98 Rev 1) on Imaging Agents. London, UK: European Medicines Agency; 2009. Available from: http://www.tga.gov.au/pdf/euguide/ewp32118008en.pdf. Accessed June 20, 2013.

2. US Department of Health and Human Services; Food and Drug Administration; Center for Drug Evaluation and Research; Center for Biologics Evaluation and Research. Guidance for Industry: Clinical Trial Endpoints for the Approval of Cancer Drugs and Biologics. Rockville, MD: Food and Drug Administration; 2007. Available from: http://www. fda.gov/downloads/Drugs/GuidanceComplianceRegulatoryInformation/ Guidances/UCM071590.pdf. Accessed June 20, 2013. 
3. US Department of Health and Human Services; Food and Drug Administration; Center for Drug Evaluation and Research; Center for Biologics Evaluation and Research. Guidance for Industry: Standards for Clinical Trial Imaging Endpoints (DRAFT Guidance). Rockville, MD: Food and Drug Administration; 2011. Available from: http://www. fda.gov/downloads/Drugs/GuidanceComplianceRegulatoryInformatio n/Guidances/UCM268555.pdf. Accessed June 20, 2013.

4. Amit O, Bushnell W, Dodd L, Roach N, Sargent D. Blinded independent central review of the progression-free survival endpoint. Oncologist 2010;15(5):492-495.

5. Walovitch RC, Chokron P, Agarwal S. US FDA draft Guidance Standard for Clinical Trial Imaging Endpoints: more than just imaging? Biomark Med. 2012;6(6):839-847.

6. US Department of Health and Human Services; Food and Drug Administration; Center for Drug Evaluation and Research; Center for Biologics Evaluation and Research. Guidance for Industry: Developing Medical Imaging Drug and Biological Products Part 3: Design, Analysis, and Interpretation of Clinical Studies. Rockville, MD: Food and Drug Administration; 2004. Available from: http://www.fda.gov/downloads/ Drugs/GuidanceComplianceRegulatoryInformation/Guidances/ ucm071604.pdf. Accessed June 20, 2013.

7. Mahaffey KW, Harrington RA, Akkerhuis M, et al; the PURSUIT Investigators. Disagreements between central clinical events committee and site investigator assessments of myocardial infarction endpoints in an international clinical trial: review of the PURSUIT study. Curr Control Trials Cardiovasc Med. 2001;2(4):187-194.

8. McGarvey LP, John M, Anderson JA, Zvarich M, Wise RA; TORCH Clinical Endpoint Committee. Ascertainment of cause-specific mortality in COPD: operations of the TORCH Clinical Endpoint Committee. Thorax. 2007;62(5):411-415.

9. Näslund U, Grip L, Fischer-Hansen J, Gundersen T, Lehto S, Wallentin L. The impact of an end-point committee in a large multicentre, randomized, placebo-controlled clinical trial: results with and without the end-point committee's final decision on end-points. Eur Heart J. 1999;20(10):771-777.

10. US Department of Health and Human Services; Food and Drug Administration; Center for Drug Evaluation and Research. Guidance for Industry: Diabetes Mellitus - Evaluating Cardiovascular Risk in New Antidiabetic Therapies to Treat Type 2 Diabetes. Rockville, MD: Food and Drug Administration; 2008. Available from: http://www.fda. gov/downloads/Drugs/GuidanceComplianceRegulatoryInformation/ Guidances/UCM071627.pdf. Accessed June 20, 2013

11. Kradjian S, Gutheil J, Baratelle AM, Einstein SG, Kaslow DC Development of a Charter for an Endpoint Assessment and Adjudication Committee. Drug Information Journal. 2005;39:53-61.

12. Food and Drug Administration. FDA Briefing Document Oncologic Drugs Advisory Committee Meeting July 24, 2012: Evaluation of Radiologic Review of Progression-free Survival in Non-hematologic Malignancies. Silver Spring, MD: Food and Drug Administration; 2012. Available from: http://www.fda.gov/downloads/AdvisoryCommittees/ CommitteesMeetingMaterials/Drugs/OncologicDrugsAdvisory Committee/UCM311141.pdf. Accessed June 20, 2013.
13. Nair R, Aggarwal R, Khanna D. Methods of formal consensus in classification/diagnostic criteria and guideline development. Semin Arthritis Rheum. 2011;41(2):95-105.

14. Jones J, Hunter D. Consensus methods for medical and health services research. BMJ. 1995;311(7001):376-380.

15. Walter SD, Cook DJ, Guyatt GH, King D, Troyan S. Outcome assessment for clinical trials: how many adjudicators do we need? Canadian Lung Oncology Group. Control Clin Trials. 1997;18(1):27-42.

16. Bolger F, Stranieri A, Wright G, Yearwood J. Does the Delphi process lead to increased accuracy in group-based judgmental forecasts or does it simply induce consensus amongst judgmental forecasters? Technol Forecast Soc Change. 2011;78(9):1671-1680.

17. Spike J, Greenlaw J. Ethics consultation: high ideals or unrealistic expectations? Ann Intern Med. 2000;133(1):55-57.

18. Janis IL. Groupthink. Psychol Today. 1971;5:43-46, 74-76.

19. Stone AM, Bushnell W, Denne J, et al; PhRMA working group. Research outcomes and recommendations for the assessment of progression in cancer clinical trials from a PhRMA working group. Eur $J$ Cancer. 2011;47(12):1763-1771.

20. Duan F, Walovitch R, Girardi V. A simulation study to evaluate accuracy and precision of blinded independent central reviews (BICR) on progression-free survival in cancer clinical trials. Joint Statistical Meeting. August 3-8, 2013; Montreal, Quebec. Available from: http:// www.amstat.org/meetings/jsm/2013/onlineprogram/AbstractDetails cfm?abstractid=309310. Accessed August 12, 2013.

21. Hong S, Schmitt N, Stone A, Denne J. Attenuation of treatment effect due to measurement variability in assessment of progression-free survival. Pharm Stat. 2012;11(5):394-402.

22. Snapinn SM. Survival analysis with uncertain endpoints. Biometrics. 1998;54(1):209-218.

23. Walovitch RC, Girardi VJ, Tomera J. Enrollment: more than numbers. Appl Clin Trials. 2012;21:30-38.

24. US Food and Drug Administration [webpage on the Internet]. Briefing information for the February 10, 2010 meeting of the Oncologic Drugs Advisory Committee. Silver Spring, MD: US Food and Drug Administration; 2010. Available from: http://www. fda.gov/advisorycommittees/committeesmeetingmaterials/drugs/ oncologicdrugsadvisorycommittee/ucm 199558.htm. Accessed June 20, 2013.

25. Herper $M$ [webpage on the Internet]. The truly staggering cost of inventing new drugs. New York, NY: Forbes.com LLC ${ }^{\mathrm{TM}}$; Feb 2012. http://www.forbes.com/sites/matthewherper/2012/02/10/the-trulystaggering-cost-of-inventing-new-drugs/. Accessed August 12, 2013.
Open Access Journal of Clinical Trials

\section{Publish your work in this journal}

The Open Access Journal of Clinical Trials is an international, peerreviewed, open access journal publishing original research, reports, editorials, reviews and commentaries on all aspects of clinical tria design, management, legal, ethical and regulatory issues, case record form design, data collection, quality assurance and data auditing

\section{Dovepress}

methodologies. The manuscript management system is completely online and includes a very quick and fair peer-review system, which is all easy to use. Visit http://www.dovepress.com/testimonials.php to read real quotes from published authors. 